\title{
Qualidade de vida das mães de crianças e adolescentes com paralisia cerebral
}

\author{
Life quality of mothers with teenagers and children victims \\ of cerebral palsy
}

\author{
Judilita Teresa de Melo Carvalho ${ }^{[\mathrm{a}]}$, Nyedja Menezes Rodrigues ${ }^{[\mathrm{b}]}$, \\ Lícia Vasconcelos Carvalho da Silva ${ }^{[\mathrm{cl}}$, Daniella Araújo de Oliveira ${ }^{[\mathrm{d}]}$
}

[a] Aluna da Associação Caruaruense de Ensino Superior (ASCES), Caruaru, PE - Brasil, e-mail: judilitateresa@yahoo.com.br

[b] Aluna da Associação Caruaruense de Ensino Superior (ASCES), Caruaru, PE - Brasil, e-mail: nynacaruaru@ig.com.br

[c] Mestre em Neuropsiquiatria e Ciências do Comportamento pela Universidade Federal de Pernambuco (UFPE), professora do curso de Fisioterapia da Associação Caruaruense de Ensino Superior (ASCES), Caruaru, PE - Brasil, e-mail: liciavcarvalho@gmail.com.br

[d] Mestre em Neuropsiquiatria e Ciências do Comportamento pela Universidade Federal de Pernambuco (UFPE), professora do curso de Fisioterapia da Associação Caruaruense de Ensino Superior (ASCES), Caruaru, PE - Brasil, e-mail: sabino_daniella@ig.com.br

\section{Resumo}

Introdução: A dependência funcional da criança com paralisia cerebral (PC) parece ocasionar uma sobrecarga física e emocional em seus familiares. Objetivo: O objetivo deste estudo foi avaliar a qualidade de vida das mães de crianças e adolescentes com PC atendidas nas clínicas de fisioterapia da rede privada de Caruaru, PE, e identificar possíveis variações de acordo com o grau de comprometimento motor e com a idade das crianças e adolescentes. Metodologia: Participaram desse estudo 31 mães de crianças e adolescentes com PC, que responderam ao questionário WHOQOL-Bref. Em seguida, os pesquisadores avaliaram o nível de função motora grossa das crianças por meio do teste Gross Motor Function Classification System (GMFCS). Resultados: $\mathrm{Na}$ análise descritiva dos dados, verificou-se que a maior parte da amostra apresentou comprometimento motor grave e idade entre 2-5 anos. Ao relacionar o grau de comprometimento motor com os domínios do questionário, observou-se que $60 \%$ das mães de crianças com comprometimento leve apresentavam uma percepção negativa do domínio físico. Quando o prejuízo motor foi moderado, a percepção da qualidade de vida materna no domínio meio ambiente foi negativa em 55,5\% da amostra. E nas mães de crianças com comprometimento motor grave, observamos uma percepção positiva nos domínios físico $(58,8 \%)$ e psicológico $(76,3 \%)$, e negativa nos domínios de relações sociais e de meio ambiente (52,6\%). Conclusão: A percepção 
da qualidade de vida de mães de crianças e adolescentes com PC na cidade de Caruaru encontra-se prejudicada, principalmente quando relacionada aos aspectos que compõem o domínio meio ambiente e das relações sociais.

Palavras-chave: Paralisia cerebral. Qualidade de vida. Crianças. Mães.

\begin{abstract}
Introduction: The functional dependency of children with cerebral palsy $(C P)$ must promote a physical and emotional stress in your parents. Objective: The objective of this study has been to evaluate life quality of mothers of teenagers and children, victims of CP assisted in Physiotherapy Clinics of the Private Enterprise in Caruaru City, PE, Brazil, and identify possible alterations on their behavior, according to the level of motor difficulty and to the age of their children and teenagers. Methodology: 31 mothers participated of this study responding to WHOCOLBref questionnaire. It was used the Gross Motor Function Classification System (GMFCS) test to evaluate the level of children's and teenagers' gross motor function. Results: Our descriptive analysis of data verified that most of the sample presented severe level of deficiency and age between 2-5 years. At linking the grade of motor affectation with the questionnaire dominium, it was observed that $60 \%$ of the mothers whose kids presented a light affectation, there was a negative perception of physical dominium. When the motor harm was soft, the perception of maternal life quality was negative in $55.5 \%$. And in mothers whose children have severe affectation, we observed a positive perception of the physical (58.8\%) and psychological (76.3\%) dominium, and a negative one at social relations and environment dominium (52.6\%). Conclusion: The perception of quality of life on mothers of children and teenagers victims of CP in Caruaru is really affected, especially in relation to the aspects which compose environment and social relations.
\end{abstract}

Keywords: Cerebral palsy. Life quality. Children. Mothers.

\title{
Introdução
}

A paralisia cerebral (PC) ou encefalopatia crônica não progressiva da infância é decorrente de uma lesão estática no encéfalo durante o período de desenvolvimento $(1,2)$. A agressão pode ocorrer no período pré, peri ou pós-natal. Assim, os principais fatores etiológicos relacionam-se a alterações circulatórias maternas, infecções, eclampsia e descolamento prematuro de placenta, prematuridade e baixo peso, icterícia grave, asfixias, parto instrumental, síndromes epilépticas, traumatismos crânio-encefálico, entre outros $(1,3)$. As manifestações clínicas nas crianças correspondem a disfunções sensório-motoras com alteração do tônus muscular, da postura e dos movimentos voluntários (2).

A incidência mundial de indivíduos com PC tem-se mantido constante nos últimos anos, acometendo 1,5 a 2,5 por mil nascidos vivos (4). A paralisia cerebral classifica-se de acordo com o tipo de tônus anormal, a distribuição do tônus anormal e o grau de prejuízo motor (5). O retardo mental, as alterações na linguagem, a disfagia, os problemas auditivos e visuais e a ocorrência de crises convulsivas podem estar associados ao prejuízo motor na paralisia cerebral, tornando a criança dependente de seus familiares para execução de atividades funcionais (5).

Fiamenghi (6) relata que o nascimento do primeiro filho de um casal está associado a uma série de mudanças no relacionamento e na unidade familiar, caracterizada pela transição nas identidades individuais de marido e mulher para pai e mãe. Frequentemente as características físicas e intelectuais das crianças são planejadas pelos pais desde o início da gravidez. Nesse período os pais almejam filhos saudáveis, inteligentes, fantasiam a carreira profissional e colocam no bebê suas expectativas e idealizações (1).

O nascimento de uma criança especial gera nos pais uma série de reações diante do inesperado, que incluem períodos de crises emocionais e de adaptações psicossociais, tornando-os susceptíveis ao 
desenvolvimento de problemas relacionados à depressão, angústia, medo, solidão, fuga e rejeição ou superproteção da criança $(6,7)$. A associação desses fatores favorece a perda da autoestima, da identidade familiar e, comumente, a separação dos pais (8).

Por outro lado, a dependência funcional da criança com paralisia cerebral, ocasiona uma sobrecarga física e emocional em seus familiares, especialmente nas mães, que assumem com frequência os cuidados com o filho (7-9). O desgaste físico e psicológico tem sido associado a prejuízos na qualidade de vida materna (7). Alguns estudos têm sido realizados com mães de crianças com PC, verificando indisposição para a realização de atividades físicas, para o relacionamento social, má percepção da saúde (10), predisposição ao estresse (11), falta de conhecimento a respeito da doença do filho, diminuição do humor e problemas no convívio social, familiar e profissional (12).

O avanço da tecnologia neonatal tem favorecido uma melhoria nos cuidados médicos perinatais e, consequentemente, a diminuição da taxa de mortalidade e o aumento da sobrevivência das crianças com idade gestacional e baixo peso cada vez mais extremos (2). A sobrevivência desses bebês está associada ao aumento na prevalência de sequelas físicas, sensoriais, cognitivas e comportamentais dessa população, e, consequentemente, ao aumento no número de familiares sobrecarregados física e emocionalmente, interferindo assim em sua qualidade de vida (5).

A Organização Mundial de Saúde (OMS) define qualidade de vida como "a percepção do indivíduo de sua posição na vida no contexto da cultura e sistema de valores nos quais ele vive e em relação aos seus objetivos, expectativas, padrões e preocupações" (13). Um dos instrumentos frequentemente utilizados na literatura para avaliação da qualidade de vida é o WHOQOL-Bref, que é aplicado em base populacional sem especificar doenças, sendo apropriado para estudos epidemiológicos. Esse instrumento de coleta de dados baseia-se na subjetividade da percepção da qualidade de vida (14).

Eker e Tuzun (15) observaram em seu estudo que o comprometimento motor da criança interfere na percepção da qualidade de vida materna. Uma das formas utilizadas para mensurar o prejuízo motor da criança com paralisia cerebral é a avaliação da função motora grossa por meio do Gross Motor Function Classification System (GMFCS), que é um sistema que tem a finalidade de fornecer uma classificação objetiva dos padrões de incapacidade motora.

Considerando que as crianças com sequelas de paralisia cerebral necessitam de assistência fisioterapêutica prolongada, uma boa relação do fisioterapeuta com a criança e sua família torna-se necessária ao alcance dos objetivos terapêuticos. Nesse sentido, o entendimento adequado da doença, das limitações funcionais e, especialmente, das potencialidades da criança possibilita maior colaboração da família, sendo essa motivação dos parentes, especialmente dos responsáveis diretos, decisiva à percepção do sucesso do tratamento.

Deste modo, o presente estudo tem como objetivo avaliar a qualidade de vida das mães de crianças e adolescentes com paralisia cerebral atendidas nas clínicas de fisioterapia da rede privada de Caruaru, PE, e identificar possíveis variações de acordo com o grau de comprometimento motor e a idade das crianças e adolescentes.

\section{Metodologia}

Participaram deste estudo 31 mães de crianças e adolescentes com paralisia cerebral que estavam em atendimento no período de maio a agosto de $2008 \mathrm{em}$ todas as clínicas de fisioterapia da rede privada de Caruaru, PE, que prestam serviços a pacientes especiais. Esta pesquisa teve início após aprovação do Comitê de Ética e Pesquisa da Associação Caruaruense de Ensino Superior (CEP/ASCES), de acordo com a Resolução 196/96 do Conselho Nacional de Saúde.

A partir de visitas às clínicas de fisioterapia da cidade, todas as mães dos pacientes foram esclarecidas a respeito dos objetivos desta pesquisa e convidadas a participar da investigação com seus filhos; entretanto, apenas 31 mães concordaram com a participação e assinaram um termo de consentimento livre e esclarecido, respondendo em seguida ao questionário WHOQOOL-Bref, para avaliação da sua qualidade de vida. O WHOQOLBref consta de 26 questões, extraídas do WHOQOL-100, sendo duas questões gerais sobre qualidade de vida e as demais distribuídas em quatro domínios: físico, psicológico, relação social e meio ambiente $(16,17)$. 
O domínio físico envolve sentimentos e expectativas quanto ao manejo da dor, desconforto pelo excesso de atividades físicas e as perturbações com o sono (18). O domínio psicológico relaciona-se aos sentimentos positivos como o aproveitar da vida, expectativas com o futuro, preocupações com as condições de saúde e os sentimentos negativos, como mau humor, ansiedade e depressão. O domínio da relação social visa a esclarecer o impacto do relacionamento social do paciente com a família, no trabalho, no lazer, sua satisfação sexual, acesso aos cuidados médicos e amparo da sociedade $(18,19,20)$. Por fim, o quarto domínio é definido como meio ambiente e avalia a sensação de segurança e conforto quanto ao ambiente em que vive o indivíduo. Fazem parte desse domínio as condições de moradia, segurança pública, recursos financeiros, acesso aos meios de transporte, educação, alimentação e lazer adequados $(20,21)$.

Em seguida à aplicação do questionário às mães, as crianças e os adolescentes foram avaliados pelos pesquisadores quanto ao nível de função motora grossa, a partir da aplicação do teste Gross Motor Function Classification System (GMFCS), que classifica o grau de incapacidade motora em cinco níveis. O nível 1 refere-se à melhor função e maior independência, e o nível 5, ao maior grau de limitação e dependência motora (16). Os pacientes são distribuídos em três grupos, conforme a gravidade do comprometimento motor: leve (GMFCS I e II), moderado (GMFCS III) e grave (GMFCS VI e V) (3).

Os critérios de exclusão adotados para essa pesquisa foram as mães que apresentavam alterações cognitivas e/ou físicas que impossibilitassem o preenchimento do questionário, e aquelas que se recusaram a assinar o termo de consentimento.

Os dados obtidos foram submetidos à análise exploratória descritiva por meio do programa estatístico SPSS $^{\circledR}$ (versão 11.5) e os resultados foram expressos em média, desvio padrão e percentual (\%).

\section{Resultados}

No período de maio a agosto de 2008, o número de crianças e adolescentes com diagnóstico de paralisia cerebral atendidos nas clínicas de fisioterapia de rede privada de Caruaru foi de 45 pacientes, entretanto apenas 31 mães concordaram em participar deste estudo.

A média de idade das crianças e adolescentes foi de 6,16 anos (6,16 \pm 0,52). Assim, 12 crianças encontravam-se na faixa etária de 2-5 anos, 10 entre 6-8 anos e 9 entre 9-12 anos. Considerando o nível de função motora grossa, 54,8\% ( $\mathrm{n}=17)$ das crianças com PC foram classificadas como níveis 4 ou 5 do GMFCS, indicando que a maioria dos pacientes apresentavam comprometimento motor grave (Tabela1).

Tabela 1 - Relação entre a idade e o comprometimento motor das crianças e adolescentes com paralisia cerebral $(\mathrm{n}=31)$

\begin{tabular}{|c|c|c|c|c|c|c|c|}
\hline \multirow[b]{3}{*}{ Idade (anos) } & \multicolumn{6}{|c|}{ Grau de comprometimento motor } & \multirow{3}{*}{$\begin{array}{c}\text { Total } \\
\%\end{array}$} \\
\hline & \multicolumn{2}{|c|}{ Leve } & \multicolumn{2}{|c|}{ Moderado } & \multicolumn{2}{|c|}{ Grave } & \\
\hline & n & $\%$ & n & $\%$ & $\mathbf{n}$ & $\%$ & \\
\hline $2-5$ & $1 / 12$ & 8,3 & $5 / 12$ & 41,7 & $6 / 12$ & 50,0 & 100,0 \\
\hline $6-8$ & $1 / 10$ & 10,0 & $4 / 10$ & 40,0 & $5 / 10$ & 50,0 & 100,0 \\
\hline $9-12$ & $3 / 9$ & 33,3 & $0 / 9$ & 0,00 & $6 / 9$ & 66,7 & 100,0 \\
\hline Total & $5 / 31$ & 16,1 & $9 / 31$ & 29,0 & $17 / 31$ & 54,8 & 100,0 \\
\hline
\end{tabular}


A Tabela 1 mostra a relação entre a idade das crianças/adolescentes e o grau de comprometimento motor; assim, na faixa etária de 9-12 anos, 33,3\% da amostra apresentou comprometimento motor leve; entre 2-5 anos, em 41,7\% dos casos o comprometimento motor foi considerado moderado; e entre 6-8 anos, 50\% apresentam comprometimento motor grave.

A análise de cada domínio demonstrou que a maioria das mães apresentava uma percepção positiva da qualidade de vida nos aspectos físico (61,3\%), psicológico (80,6\%) e relações sociais (58,1\%). Entretanto, no domínio meio ambiente 51,6\% das mães referem uma percepção negativa da sua vida, independentemente da idade das crianças e do grau de comprometimento motor (Tabela 2).

Tabela 2 - Percepção materna dos domínios físico, psicológico, relação social e meio ambiente, de acordo com o grau de comprometimento motor $(\mathrm{n}=31)$

\begin{tabular}{|c|c|c|c|c|c|c|c|c|c|}
\hline \multirow{3}{*}{$\begin{array}{l}\text { Comprometimento } \\
\text { motor }\end{array}$} & \multirow{3}{*}{$\mathrm{n}$} & \multicolumn{2}{|c|}{ Físico } & \multicolumn{2}{|c|}{ Psicológico } & \multicolumn{2}{|c|}{ Rel. Sociais } & \multicolumn{2}{|c|}{ Meio Ambiente } \\
\hline & & \multicolumn{2}{|c|}{ Percepção } & \multicolumn{2}{|c|}{ Percepção } & \multicolumn{2}{|c|}{ Percepção } & \multicolumn{2}{|c|}{ Percepção } \\
\hline & & positiva & negativa & positiva & negativa & positiva & negativa & positiva & negativa \\
\hline Leve & 5 & $40 \%$ & $60 \%$ & $100 \%$ & $0 \%$ & $60 \%$ & $40 \%$ & $60 \%$ & $40 \%$ \\
\hline Moderado & 9 & $77,8 \%$ & $22,2 \%$ & $77,8 \%$ & $22,2 \%$ & $77,8 \%$ & $22,2 \%$ & $44,4 \%$ & $55,6 \%$ \\
\hline Grave & 17 & $58,8 \%$ & $41,2 \%$ & $76,5 \%$ & $23,5 \%$ & $47,1 \%$ & $52,9 \%$ & $47,1 \%$ & $52,9 \%$ \\
\hline
\end{tabular}

Ao relacionar o comprometimento motor com os domínios físico, psicológico, relações sociais e de meio ambiente (Tabela 2), verificou-se que 60\% das mães de crianças com prejuízo motor leve apresentavam uma percepção negativa do domínio físico, e positiva dos demais domínios.

Quando o grau de comprometimento motor da criança era moderado, havia uma percepção positiva nos domínios físico, psicológico e de relações sociais em 77,8\% das mães; contudo, a percepção negativa dos aspectos relacionados ao meio ambiente foi referida por 55,6\% dessas mães. Considerando o déficit motor grave, a maioria das mães demonstrou uma percepção positiva nos domínio físico $(58,8 \%)$ e psicológico (76,5\%), enquanto as relações sociais e o meio ambiente são percebidos negativamente por $52,9 \%$ das mães.

Tabela 3 - Percepção materna dos domínios físico, psicológico, relação social e meio ambiente, de acordo com a idade das crianças e adolescente $(\mathrm{n}=31)$

\begin{tabular}{|c|c|c|c|c|c|c|c|c|c|}
\hline \multirow[b]{3}{*}{ Idade (anos) } & \multirow[b]{3}{*}{$\mathrm{n}$} & \multicolumn{2}{|c|}{ Físico } & \multicolumn{2}{|c|}{ Psicológico } & \multicolumn{2}{|c|}{ Rel. Sociais } & \multicolumn{2}{|c|}{ Meio Ambiente } \\
\hline & & \multicolumn{2}{|c|}{ Percepção } & \multicolumn{2}{|c|}{ Percepção } & \multicolumn{2}{|c|}{ Percepção } & \multicolumn{2}{|c|}{ Percepção } \\
\hline & & positiva & negativa & positiva & negativa & positiva & negativa & positiva & negativa \\
\hline $2-5$ & 12 & $66,7 \%$ & $33,3 \%$ & $83,3 \%$ & $16,7 \%$ & $66,7 \%$ & $33,3 \%$ & $58,3 \%$ & $41,7 \%$ \\
\hline $6-8$ & 10 & $80 \%$ & $20 \%$ & $90 \%$ & $10 \%$ & $70 \%$ & $30 \%$ & $60 \%$ & $40 \%$ \\
\hline $9-12$ & 9 & $33,3 \%$ & $66,7 \%$ & $66,7 \%$ & $33,3 \%$ & $33,3 \%$ & $66,7 \%$ & $22,2 \%$ & $77,8 \%$ \\
\hline
\end{tabular}


A Tabela 3 demonstra a relação entre a idade dos filhos e a percepção materna sobre o domínio físico, psicológico, relação social e meio ambiente. Foi observada uma percepção positiva de todos os domínios pelas mães que possuem filhos com idade entre 2-5 anos e 6-8 anos. Quando a faixa etária dos filhos foi de 9-12 anos, as mães relataram uma percepção negativa dos domínios físicos, relações sociais e de meio ambiente, sugerindo que a idade da criança poderia modificar a percepção da qualidade de vida das mães. Entretanto, nenhuma associação estatística foi estabelecida.

\section{Discussão}

A expressão "qualidade de vida" engloba vários significados e diz respeito à maneira como as pessoas vivem, sentem e compreendem o seu cotidiano (18). Os elementos que incluem a avaliação da qualidade de vida estão relacionados aos aspectos culturais, históricos, de classes sociais, ao conjunto de condições materiais e não materiais, diferenças por faixas etárias e condições de saúde das pessoas ou comunidade.

Vários instrumentos para a avaliação da qualidade de vida foram desenvolvidos, os quais levam em consideração a ênfase na subjetividade, a amplitude de aspectos investigados e a apreciação do paciente como indivíduo único $(14,19)$.

A análise dos resultados relacionados à percepção da qualidade de vida materna no domínio físico apontou para um desgaste físico materno mais evidente quando as crianças encontravam-se numa faixa etária de 9 a 12 anos. Estudos realizados (10-12,22-24) verificaram que os cuidados prestados a uma criança com desordens neurológicas reduzem os níveis de saúde física materna. Sousa e Pires (25) afirmam que o desgaste físico materno é sempre elevado, independentemente do grau de cuidados e atenção prestados aos seus filhos.

De acordo com a literatura, o desgaste físico dos pais de crianças com sequelas neurológicas atinge altos níveis e pode ser responsável também por uma percepção negativa da qualidade de vida de seus filhos $(9,26,27)$.

Mães e crianças podem ter percepções diferentes do impacto de doenças na qualidade de vida destas últimas(26). Por outro lado, essa percepção de que os filhos estão sofrendo em consequência de doenças ou limitações funcionais pode ser um fator responsável pela elevação do estresse físico vivenciado pelas mães.

Pesquisa dos prejuízos na qualidade de vida de pais de crianças e adolescentes com desordens do desenvolvimento evidenciou uma percepção negativa nos domínios físico e emocional, de relações sociais e de meio ambiente nos pais de crianças com paralisia cerebral, retardo mental ou autismo, quando comparado aos pais de crianças saudáveis (10).

Eker e Tuzun (15), em seu estudo utilizando o questionário SF-36, verificaram uma diminuição de todas as escalas do instrumento de avaliação de qualidade de vida. $\mathrm{Na}$ análise dos dados do grau de comprometimento motor das crianças observou-se maior redução da saúde física nas mães de crianças com paralisia cerebral que apresentavam comprometimento motor mais expressivo.

Uma avaliação da qualidade de vida de 818 crianças com PC a partir dos relatos dos pais ressaltou que o grau de incapacidade funcional nem sempre está associado à percepção negativa da qualidade de vida, já que a percepção da aceitação social, do ambiente escolar e de fatores emocionais poderia estar mais prejudicada em crianças com menor prejuízo motor (28), em concordância com os resultados evidenciados no estudo atual. Por outro lado, a presença de dor parece ser um fator limitante na qualidade de vida dessas crianças (29).

Ao analisarmos o domínio psicológico, constatamos uma percepção positiva da qualidade de vida materna em nosso estudo. Esses resultados não correlacionam com os encontrados na literatura, que relatam que as mães de crianças com patologias neurológicas apresentam maior predisposição para o desenvolvimento de psicopatologias, tais como ansiedade, estresse e depressão $(10,15,22-25,30)$.

Observa-se ainda que as experiências vivenciadas pelas mães de crianças com paralisia cerebral remetem a uma série de reações que incluem períodos de crises emocionais e de adaptações, relacionadas à 
sobrevivência da criança, seu desenvolvimento, integração na sociedade e sua autonomia, caracterizando a preocupação com o futuro como fator de maior influência no declínio psicológico das mães (25).

No domínio das relações sociais, verificamos na pesquisa que a percepção da qualidade de vida materna nesse domínio encontrava-se negativa quando as crianças enquadravam-se na faixa etária de 9 a 12 anos e com comprometimento motor grave.

Estudos encontrados $(15,25,30)$ apontam que ocorre diminuição do apoio prestado pela sociedade e familiares às mães de crianças portadoras de disfunções neurológicas, por elas serem isoladas socialmente. De acordo com Eker e Tuzun (15), as reduções nos valores que constituem o domínio das relações sociais identificam a dimensão da interferência do desgaste físico e emocional no convívio social.

Segundo Lima (7), quando as mães têm um bom relacionamento e apoio familiar, elas se apresentam mais disponíveis e com menor sobrecarga emocional, independente do grau de comprometimento motor da criança. A mesma autora relata que o apoio materno reflete na diminuição do desenvolvimento de alguns problemas de saúde para a mãe e no auxílio aos cuidados com as crianças.

Os resultados da análise do domínio meio ambiente em nossa pesquisa revelaram um prejuízo na percepção da qualidade de vida materna quando as crianças apresentavam idade entre 9 a 12 anos e comprometimento motor moderado ou grave.

Monteiro, Matos e Coelho (30) referem que maiores níveis econômicos estão relacionados a uma melhor satisfação com a vida. O mesmo estudo descreve que as pesquisas que comprovam esta relação foram realizadas em países em desenvolvimento, os quais ainda não apresentam adequada infraestrutura para atender às necessidades das crianças com paralisia cerebral. Apontam ainda que nas famílias que residem nos bairros periféricos a qualidade de vida materna é inferior. Em nosso estudo observamos que as mães que apresentavam níveis socioeconômicos menores, a qualidade de vida encontrava-se reduzida. A maior interferência na percepção da qualidade de vida relatada pelas mães associa-se às dificuldades no acesso a transporte, às condições de infraestrutura do bairro onde residem e à precariedade da assistência e cuidados médicos.

Lima (7) constatou que a maioria das famílias de crianças com PC possui um nível socioeconômico reduzido, o que acentua ainda mais os problemas físicos das mães, em virtude das dificuldades de manuseio das crianças decorrentes das condições de moradia restritas, dificuldades no acesso aos meios de transporte público, interferindo na socialização das crianças e adolescentes.

\section{Conclusão}

Com base nos dados obtidos, este estudo demonstrou que a percepção da qualidade de vida das mães de crianças e adolescentes com paralisia cerebral na cidade de Caruaru, PE, apresenta-se prejudicada, principalmente quando relacionada aos fatores que compõem os aspectos de relações sociais e de meio ambiente, em que o comprometimento motor grave e a idade elevada das crianças parecem interferir na redução da percepção da qualidade de vida materna.

Nesse sentido, os fatores como a inclusão social e o acesso aos serviços, como saúde, escola, lazer, entre outros, parecem funcionar como barreiras para as famílias de crianças com paralisia cerebral.

Os resultados deste estudo chamam a atenção para a necessidade de melhoramento nas condições de acessibilidade e de maior aceitação da sociedade quanto às diferenças existentes entre as pessoas. Por outro lado, ressalta a necessidade de participação de fisioterapeutas nesse processo enquanto profissionais que atuam na promoção de saúde.

Sugere-se, entretanto, que novos estudos sejam realizados nesta área, utilizando uma amostra mais ampla visando a acrescentar informações ao corpo de conhecimento da fisioterapia.

\section{Referências}

1. Rotta NT. Paralisia cerebral, novas perspectivas terapêuticas. J Pediatr. 2002;78(Supl1):48-54. 
2. Mancinni MC, Fiuza PM, Rebelo JM, Magalhães LC, Coelho ZAC, Paixão ML, et al. Comparação do desempenho de atividades funcionais com desenvolvimento normal e crianças com Paralisia Cerebral. Arq Neuropsiquiatr. 2002;60(2-B):446-52.

3. Morales NMO. Avaliação transversal da qualidade de vida em crianças e adolescentes com paralisia cerebral por meio de um instrumento genérico (CHQ-PF50) [dissertação]. Uberlândia: Faculdade de Medicina, Universidade Federal de Uberlândia; 2005.

4. Leite JMRS, Prado GF. Paralisia cerebral: aspectos fisioterapêuticos e clínicos. Revista Neurociências. [online]. 2004 [acesso 2 dez. 2008];12(1). Disponível em: http://www.unifesp.br/dneuro/neurociencias/vol12_1/paralisia_cerebral.htm

5. Freitas PM, Carvalho RCL, Leite MRS, Haase VG. Relação entre o estresse materno e a inclusão escolar de crianças com paralisia cerebral. Arq Bras Psicol. 2005;57(1):46-57.

6. Fiamenghi JR, Messa A. Pais, filhos e deficiência: estudos sobre as relações familiares. Psicol Ciênc Prof. 2007;27(2):236-45.

7. Lima RABC. Envolvimento materno no tratamento fisioterapêutico de crianças portadoras de deficiências: compreendendo dificuldades e facilitadores [dissertação]. Belo Horizonte: Faculdade de Medicina, Universidade Federal de Minas Gerais; 2006.

8. Silveira MLM, Giorge AH, Furuta EA. Contribuição do pediatra para a qualidade de vida na constituição da família. In: Diniz DP, Schor N. Guia de qualidade de vida. Barueri: Manole; 2006, p. 133-37.

9. White-Koning M, Grangjean H, Colver A, Arnaud C. Parent and professional reports of the quality of life of children with cerebral palsy and associated intellectual impairment. Dev Med Child Neurol. 2008;50(8):618-24.

10. Mugno D, Ruta L, D’Arrigo V, Mazzone L. Impairment of quality of life in parents of children and adolescents with pervasive developmental disorder. Health Qual Life Outcomes. 2007;5:22.

11. Sen K, Yurtsever S. Difficulties experienced by families with disabled children. J Spec Pediatr Nurs. 2007;12(4):238-52.

12. Ones K, Yilmaz E, Cetinkaya B, Caglar N. Assessment of the quality of life of mothers of children with cerebral palsy (primary caregivers). Neurorehabil Neural Repair. 2005;19(3):232-7.

13. Gração DC, Santos MGM. A percepção materna sobre a paralisia cerebral no cenário da orientação familiar. Fisioter Mov. 2008;21(2):107-13.

14. Minayo MCS, Hartz MA, Buss PM. Qualidade de vida e saúde: um debate necessário. Ciênc Saúde Coletiva. 2000;5(1):7-18.

15. Eker L, Tuzun EH. An evaluation of quality of life of mothers of children with cerebral palsy. Disabil Rehabil. 2004;26(23):1354-9.

16. Cristofolleti G, Hygashi F, Godoy ALR. Paralisia cerebral: uma análise do comprometimento motor sobre a qualidade de vida. Fisioter Mov. 2007;20(1):37-44.

17. Fleck MPA, Leal OF, Louzada S, Xavier M, Chachamovich E, Vieira G, et al. Desenvolvimento da versão em português do instrumento de avaliação de qualidade de vida da OMS (WHOQOL - 100). Rev Bras Psiquiatr. 1999;21(1)40-6.

18. Gonçalves A, Vilarta R. Qualidade de vida: concepções básicas voltadas à saúde. In: Gonçalves A, Vilarta R. Qualidade de vida e atividade física: explorando teoria e prática. Barueri, SP: Manole; 2004. p. 27-62.

19. Belasso AGS, Sesso RCC. Qualidade de vida: principais focos de estudo e intervenções. In: Diniz DP, Schor N. Guia de qualidade de vida. Barueri: Manole; 2006. p. 1-10. 
20. Duarte OS, Ciconelli RM. Instrumentos para avaliar a qualidade de vida: genéricos e específicos. In: Diniz DP, Schor N. Guia de qualidade de vida. Barueri: Manole; 2006. p. 11-8.

21. Neri AL. Qualidade de vida na velhice. In: Rebellato JR, Morelli JGS. Fisioterapia geriátrica: a prática da assistência ao idoso. Barueri, SP: Manole; 2004. p. 1-36.

22. Manuel J, Naughton MJ, Balkrishnan R, Smith BP, Koman LA. Stress and adaptation in mothers of children with Cerebral Palsy. J Pediatr Psychol. 2003;28(3):197-201.

23. Barbosa MAM, Chaud MN, Gomes MMF. Vivências de mães com um filho deficiente: um estudo fenomenológico. Acta Paul Enferm. 2008;21(1):46-52.

24. Sunelaitis RC, Arruda DC, Marcom SS. A repercussão de um diagnóstico de Síndrome de Down no cotidiano familiar: perspectiva da mãe. Acta Paul Enferm. 2007;20(3):264-71.

25. Sousa SCB, Pires AAP. Comportamento materno em situação de risco: mães de crianças com paralisia cerebral. Psicologia, saúde e doenças. Psicologia, Saúde \& Doenças. 2003;4(1):111-30.

26. Kiss E, Kapornai K, Baji I, Mayer L, Vetró A. Assessing quality of life: mother- child agreement in depressed and non-depressed Hungarian. Eur Child Adolesc Psychiatry. 2009;18(5):265-73.

27. Cremeens J, Eiser C, Blades M. Factors influencing agreement between child self-report and parent proxy-reports on the Pediatric Quality of Life Inventory 4.0 (PedsQL ${ }^{\mathrm{TM}}$ ) generic core scales. Health Qual Life Outcomes. 2006;4:58.

28. Arnaud C, White-Koning M, Michelsen SI, Parkes J, Parkinson K, Thyen U, et al. Parent-reported quality of life of children with cerebral palsy in Europe. Pediatrics. 2008;121(1):54-64.

29. Dickinson HO, Parkinson KN, Ravens-Sieberer U, Schirripa G, Thyen U, Arnaud C et al. Self-reported quality of life of 8-12-year-old children with cerebral palsy: a cross-sectional European study. Lancet. 2007;369(9580):2171-8.

30. Monteiro M, Matos AP, Coelho R. Adaptação psicológica de mães cujos filhos apresentam paralisia cerebral: resultados de um estudo. Rev Port Psicossom. 2004;6(1):115-30.

Recebido: $17 / 12 / 2008$

Received: $12 / 17 / 2008$

Aprovado: 07/05/2010 Approved: 05/07/2010

Revisado: 23/06/2010

Reviewed: 06/23/2010 